Practice Guidelines in Primary Care 2002. By Ralph Gonzales and Jean S. Kutner. 181 pp. New York, McGraw Hill Medical, 2002. \$14.95 (paper). ISBN 0-07-137797-2.

This palm-sized paperback reference offers a host of primary care guidelines in the areas of disease screening, disease prevention, and disease management. An annual update, this edition documents each topic with recent major, national organization recommendations or evidence-based citations.

Disease screening addresses the standard concerns in primary care, such as screening for cancer, hyperlipidemia, obesity, osteoporosis, alcohol abuse, and depression. Each area has a table comparing opinions from various authorities (US Public Service Task Force, American Cancer Society, American Academy of Family Physicians [AAFP]) as well as notes defining special circumstances and added commentary.

Disease prevention is primarily concerned with immunizations, featuring American College of Physicians and AAFP recommendations (includes varicella and pneumococcal vaccines). Additional reviews discuss cancer, myocardial infarction, osteoporosis, and stroke prevention methods. Often algorithms provide the reader with a concise presentation of specific disease prevention.

Disease management guidelines prove to be the most practical aspect of the book, with evidenced-based recommendations for such disorders as atrial fibrillation, asthma, congestive heart failure, depression, diabetes, hypertension, low back pain, hormone replacement therapy, and urinary tract infections. Excellent algorithms are used, and each management guideline is described succinctly and with superb documentation. These are stateof-the-art clinical guidelines for patient care decision making.

The Appendix gives the clinician quick access to the standard screening tools for depression, alcohol abuse, and dementia, and cognitive dysfunction.

There is a strong emphasis on evidence-based clinical pathways and guidelines in current medical practice, and this compact reference proves extremely helpful in dayto-day office practice. The clinician who relies on such pathways in patient care cannot help but appreciate the efficacy of this guide.

James J. Bergman, MD Bellevue, Wash

Interpreting the Medical Literature. 4th edition. By Stephen H. Geblbach. 296pp. New York, McGraw Hill Medical, 2002. \$34.95 (paper). ISBN 0-07-138762-5.

I have been struggling lately to teach family practice residents about the principles of evidence-based medicine, the process of searching the literature, and the value of reading original research articles. That is why Gehlbach's Interpreting the Medical Literature is timely and such a pleasure to read. Now in its fourth edition, this book continues to be a well-written and definitive guide to reading and understanding original research articles in the medical literature. It offers in-depth analyses of common study designs, a clear explanation of statistical significance, and an excellent chapter on interpreting basic statistical tests and regression-refreshingly done without a single mathematical formula!

The book itself is logically organized, beginning with chapters on various types of study designs, moving to analysis, and then interpretation. The chapters on interpretation include excellent discussions of bias, risk, and measurement. One highlight of the book is the terrific collection of examples from the medical literature that illustrate the book's many lessons. These classic articles range from the original case-control studies of toxic shock syndrome to the Nurses' Health Study. There is little to quibble about in this book, except perhaps to point out the wretched fact that, even with the best interpretation of available medical literature, many clinical decisions and most health policy decisions are made today without regard to the evidence at hand-the mammography controversy and prostate-specific antigen testing come to mind.

Interpreting the Medical Literature is a fine guide to the fundamental core of evidence-based medicine, the ability to read and correctly understand the medical literature. This skill is not simply about wordmanship or being able to debate the lead article in $7 A M A$. Learning and teaching how to interpret the medical literature are critical to the success of family medicine in the 21 st century. I, for one, am grateful for this book. It has helped me explain to my residents why being a good family physician means more than reading abstracts and review articles.

Frederick Chen, $\mathrm{MD}$

Center for Primary Care Research

Rockville, Md

Nonsurgical Sports Medicine-Preparticipation Exam Through Rehabilitation. By N. Nichole Barry, Michael F. Dillingham, and James L. McGuire. 336 pp, illustrated. Baltimore, Johns Hopkins University Press, 2002. \$28.50 (paper). ISBN 0-8018-5896-3.

The authors of Nonsurgical Sports Medicine stress that "non-operative sports medicine has reached proportions unimaginable just a decade ago." Underscoring their point is the dramatic increase in journals and textbooks related to primary care sports medicine. The relevance of this area to family physicians continues to increase as evidence that physical fitness leads to better overall health and longevity appears in the medical literature. 
This text is organized into 12 relatively brief chapters beginning with the preparticipation physical examination. The next sections discuss common medical conditions, including ergogenic aids. The authors then review common injuries to the head, spine, upper extremity, and lower extremity and provide an overview of overuse problems. The final section of the text addresses special situations, such as older and younger athletes, female athletes, environmental conditions, and medical coverage of events. Supplementing this information are a number of useful tables. Most illustrations are simple drawings, and no photographs or detailed illustrations are included.

Unfortunately, although the authors applaud the advances in nonsurgical sports medicine, and the cover of the book advertises this guide as comprehensive, the text falls short of reaching this goal. The authors cannot produce a comprehensive text in a brief publication. In this sense, the discussion in most chapters occurs at a depth appropriate for medical students or practitioners with limited experience in sports medicine and orthopedics. The experienced family physician or physician who emphasizes primary care sports medicine would find little new information.

References are also weak, and many chapters do not have updated information from recent literature. The preparticipation examination chapter, for example, should include the preparticipation monograph endorsed by most major medical societies. Current National Collegiate Athletic Association regulations regarding participation with illnesses and infectious diseases and newer treatment strategies as they relate to sports medicine would add to the text. Another example is the section on diabetes, which does make a number of good points, but it should guide physicians to use newer medications in the treatment of the athletic patient. The section on exercise-induced asthma addresses concerns of patients who have classic exercise-induced asthma but not those who would need a greater focus on anti-inflammatory medications as a part of their treatment regimen. Chapters would also be improved by inclusion of clear returnto-play guidelines for the various illnesses, injuries, and topics discussed in the text.

In summary, this brief volume, authored by experienced sports medicine physicians, might have a useful role in introducing medical students and inexperienced physicians to the area of nonsurgical sports medicine. The paperback is affordable, and the chapters are readable and concise. Experienced physicians, however, would likely choose from the more comprehensive textbooks that already exist in the market.

Karl B. Fields, MD

Family Medicine Residency Program Greensboro, NC

21st Edition Rudolph's Pediatrics. By Colin D. Rudolph, Abraham M. Rudolph, Margaret K. Hostetter, George Lister, and Norman J. Siegel, editors. 2,688 pp. New York, McGraw Hill Medical, 2003. \$130. ISBN 0-8386-8285-0.
This authoritative textbook is a recognized standard in the field of pediatrics, and this 21st edition updates the previous version, published in 1996. Colin P. Rudolph has assumed the position of senior editor from his father, Abraham M. Rudolph, and has enlisted several other associate editors, as well as 495 individual contributors selected as authorities in their respective pediatric subspecialties. Ideally, a textbook is a compendium of the total current state of knowledge in a specific discipline or field of study. From the outset, this task is impossible. The size of the final product forces some constraints on the content, and the time elapsed between writing and publication ensures that some of the newest developments might not be included. The editors must strike a balance not only between thoroughness, size, rapidity of new developments, and costs of new editions but also between basic science and clinically applicable content. This new edition appears to have accomplished these goals.

Although this edition adheres to the basic style and format of its predecessor, it has some notable changes. The growth of pediatric knowledge is reflected in the increase of 351 pages, 170 contributors, and a whopping $41 / 2$ pounds. Weighing in at 13 pounds, this book is definitely a reference text, not bedside handbook. Most sections have been revised and references have been updated since the previous edition. The bulk of the chapters remain organized by organ system, but new additions include sections on the acutely ill child, rehabilitation, and diagnostic techniques. These additions are beneficial. The section of the acutely ill child is weighted heavily toward basic physiology and is not simply a clinical cookbook.

The rehabilitation section includes such practical information as tracheostomy care, a selection of orthopedic appliances, and issues relating to the family's adaptation to chronic illness. Much of this information is difficult to locate from other sources. The diagnostic technology chapter discusses advances in DNA testing, biochemical screening, and the gamut of new imaging studies, as well as some analysis of statistical reporting. Other revisions from the previous edition include a doubling of the section on asthma, with inclusion of the new National Institutes of Health guidelines; advances in neonatal care, such as the use of inhaled nitric oxide; a completely revised genetics section; and many others.

One notable change for the worse is in the Table of Contents. The previous edition and the primary competition text both have subheadings in the Table of Contents that make it easy to locate a specific topic. This version simply lists the chapter titles, so that to locate a specific item quickly, the reader must resort to the index. Two other deletions from the previous edition are the chapters on drug distribution and therapy and the medication tables previously included in the appendix.

This excellent reference is for both students and clinicians seeking an authoritative and comprehensive textbook on pediatrics. It is current and complete, as well as readable. Compared with some of the more clinically oriented textbooks, this one includes a thorough discus- 
sion of the basic science aspect of various conditions while it retains clinically useful information, such as diagnostic and therapeutic algorithms. The chapter on developmental and behavioral pediatrics continues to make available the basics of office-based pediatric practice by including the information necessary to counsel patients on everyday issues, such as colic, sleep disorders, and attention-deficit hyperactivity disorder. Each section has many charts and tables and current references.

For those who own the previous edition, the passage of 6 years and the extensive revisions in this new edition would make investing in this upgrade worthwhile. The size is imposing, and I suspect that future editions will grow to two volumes.

Leland Davis, MD

University of California, San Francisco

\section{Blackwell's Primary Care Essentials: The Complete Guide. Fourth edition. And Guidelines for Electronic. $B y$ Daniel K. Onion. 882 pp. Malden, Mass, Blackwell, 2003. \$42.95 (paper). ISBN 0-632-04633-3.}

Pocket-sized primary care reference books have long been a source of clinical information to the medical student, resident physician, and practicing clinician. With the increasing popularity of personal digital assistants (PDAs), the electronic versions of these resources allow for efficient retrieval. Blackwell's Primary Care Essentials, published in both a paper and electronic format, is in its fourth edition and written nearly exclusively by Dr. Onion. The electronic version retains the original title of The Little Black Book for Primary Care, available for both the Palm and Windows CE operating systems.

The 880-page paper publication is presented as a ready source of common primary care information to the student, resident, and clinician. An intended purpose, stated in the preface, is to serve the student and resident as a starter notebook, and it does provide solid, basic information on the common conditions seen in general practice.

I liked the evidenced-based format, with ready references within the text of the clinical topic. Unfortunately, the references are contemporary only until 2001, and some were more than 20 years old. Although organization is user friendly, I found the text often too cryptic in the author's attempt to reduce the book's size to keep it portable. The book is still a bit bulky $(5 \times 7 \times 1.5$ inches $)$ to be truly portable.

The electronic version, The Little Black Book, is worthy of consideration as an addition to a PDA. I loaded a full version on my Compaq IPAQ (Windows CE version) and found the information to retrieve and the organization intuitive.

I recommend Blackwell's Primary Care Essentials as a sound choice for a portable reference for the general content of family practice. The electronic version is the most portable and is my preference for those students, residents, and practicing clinicians seeking a ready source of clinical information.

Keith A. Frey, MD, MBA

Mayo Clinic Scottsdale

Scottsdale, Ariz

Informatics in Primary Care: Strategies in Information Management for the Healthcare Provider. By Thomas E. Norris, Sherrilynne S. Fuller, Harold I. Goldberg, and Peter Tarczy-Hornoch. 241 pp. New York, Springer-Verlag, 2002. $\$ 39.95$ (paper). ISBN 0-387-95333-7.

This book was written to address the needs of primary care physicians in understanding the challenges and promises of the developing science of medical informatics. The computerized medical record is only the starting point for the electronic system, which can and should augment the medical care provided in the physicianpatient relationship. Entering and tracking orders, data management, links to laboratory results and imaging, real-time use of knowledge resources and decisionsupport tools, and care management tools, such as health maintenance, alerts, and patient notification, are all part of the ideal integrated system. In the new environment of patient-physician partnership, there will be increased emphasis on patient access to the computerized medical record and knowledge base as well as electronic communications between the partners.

The separate chapters of this book are written by different authors who have a wide range of expertise. The first five chapters offer an excellent introduction to the technical aspects of the computerized medical record and the importation of data from other sources, such as other programs and databases, laboratory data, and images. Chapter 3, by James I. Hoath, contains a superb discussion of the HL7 interface and standards for exchanging information between various systems of health information.

Chapters 6 through 10 cover the usefulness of the computerized medical record for incorporating evidence-based medicine, clinical decision support, knowledge resources, patient education, and workflow automation. Chapter 13 describes the role of the computerized medical record in quality improvement through reporting and practice analysis.

The remaining chapters discuss electronic billing, privacy and security issues, and telecommunications in primary care.

Physicians in small offices or groups do not have the advantage of having an information technology department and therefore must amass a level of knowledge sufficient to acquire, implement, and utilize their electronic systems with the help of outside resources. Larger institutions need the advice and expertise of clinicians who understand both the clinical and the technical parameters for developing an expert computer information system.

This book gives a superb introduction to medical informatics for the primary care physician. It is recommended for anyone involved with introducing comput- 
erization to a small office, clinic, or larger medical institution.

David M. Gimlett, MD Edmonds, Wash

Cosmetic Dermatology Principles and Practice. By Leslie Baumann. 266 pp, illustrated. New York, McGraw Hill Medical, 2002. \$149.00. ISBN 0-07-136281-9.

The author of this book is affiliated with the Division of Cosmetic Dermatology at the University of Miami in Florida. When one reads the preface of Cosmetic Dermatology Principles and Practice, the author's motivations for writing the text become clear, and they are, indeed, admirable. Disillusioned by the dearth of well-done published data in the realm of cosmetic dermatology, she has attempted to compile in textbook fashion all the available information on various cosmetic products, procedures, and problems. Among the multiple reasons published data and research are lacking in this field is that cosmetics are not classified as drugs and are therefore not subject to Food and Drug Administration testing and approval. The result is a minimal amount of good evidence-based data on the effectiveness and safety of products that make up a multi-billion dollar industry. Up to now, much of the information with which physicians and aestheticians have to work is provided by industry sales representatives.

With this definitive textbook, Baumann attempts to bridge the gaps and foster productive working relationships among physicians, cosmetic chemists, and aestheticians-all of whom would find this book useful. She envisions developing this field of medicine by encouraging sound scientific research, data collection, and publications, and she has done an excellent job of sifting through the available knowledge and presenting it in an organized fashion. Because the book is written for an audience of cosmetic dermatologists, it lacks relevance for most busy family physicians. Even so, however, select chapters do serve as an excellent resource for common dermatologic problems seen by family physicians, such as acne and hair loss.

Cosmetic Dermatology is well written. It is easy to read, does not bog down in lengthy sentences and explanations, and is a practical guide for its target audience. The book has many excellent photographs and well-drawn illustrations. It is organized in a logical manner, which is evident from the Table of Contents. Part 1 addresses the basic concepts of skin science and contains such chapters as photoaging, cigarettes and aging skin, dry skin, and hair science and hair loss. Part 2 addresses skin disease and contains chapters on acne and pigment disorders. Both part 1 and 2 have several chapters that would be useful to a family physician. Part 3, on cosmetic science, delves into the chemical basis behind how many products work. Part 4 addresses cosmetic dermatologic procedures such as Botox, collagen injections, chemical peels, and laser hair removal-probably not procedures done in most family physicians' offices. Part 5 takes a broader look at cosmetic dermatology and addresses some of the issues of drugs versus cosmetics in more depth. There is also a fascinating chapter on the psychosocial aspects of cosmetic dermatology written by Edmund Weisberg, Baumann's managing editor.

In summary, Cosmetic Dermatology Principles and Practice does achieve what Dr. Baumann had hoped. Targeted at cosmetic dermatologists, it is a well-organized, practical guide to a previously nebulous field of medical knowledge that was largely driven by sales representatives. Her hope to apply a scientific method to this field of medicine is a goal to be commended. Because of the intended audience, this book is not applicable to the practice of most family physicians.

Tamara Buchel, MD Mayo Thunderbird Scottsdale, Ariz 